\title{
On Gravity Currents in Heterogeneous Porous Media
}

\author{
Daniel M. Anderson ${ }^{\mathrm{a} *}$, Richard M. McLaughlin ${ }^{\mathrm{b}} \dagger$, and Cass T. Miller ${ }^{\ddagger}$

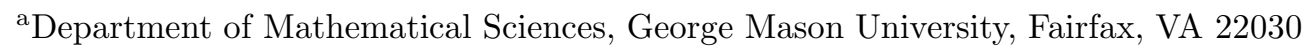 \\ ${ }^{\mathrm{b}}$ Department of Mathematics, University of North Carolina, Chapel Hill, NC, 27599 \\ ${ }^{c}$ Department of Environmental Sciences and Engineering, University of North Carolina, Chapel Hill, \\ NC, 27599
}

We examine the case of density-dependent flow in heterogeneous porous medium systems bounded by a free surface using homogenization methods for leading-order approximations. Specifically we consider the two-dimensional case in which variations occur in both the horizontal and vertical directions. Such problems lead to the need to solve cell problems to compute the solution, which is generally done using numerical approaches. We review the general homogenization results for general topology and aspect ratio. We derive an analytical solution for a case with two-dimensional variability in the slender limit for certain assumed scaling of the permeability, and we find excellent agreement with the numerical solution. We also consider the case of two miscible fluids with an assumed sharp interface and contrasting densities. We derive an analytical solution in a thin limit neglecting mixing and show agreement with known solutions for limiting cases.

\section{INTRODUCTION}

Density dependent flow in porous media is both scientifically stimulating and of significant practical interest. For example, Miller and workers have recently suggested a novel class of remediation methods based upon the injection of a dense brine, which is used to restrict the movement of dense non-aqueous phase liquids and aid remediation [1-3]. For such systems, the behavior of the brine as a function of space and time is a critical issue. Since natural porous medium systems are typically heterogeneous, the behavior of brines under such conditions is of central interest.

In previous work [4], hereafter referred to as AMM, we have examined the use of homogenization methods for calculating leading-order approximations and first-order corrections to determine the time evolution of the free surface of a brine in porous media that are heterogeneous in the vertical direction, the horizontal direction, and in both the vertical and horizontal dimensions. Both analytical similarity and numerical solutions were derived in certain limits. However, the two-dimensional work performed to date was only preliminary in nature. Further, systems of primary concern will have a brine fluid contained within a freshwater zone, leading to two-fluid problems, which have not been considered to date using homogenization methods to the best of our knowledge.

The overall goal of this work is to improve our understanding of the dynamics of dense brines in heterogeneous porous medium systems. The specific objectives of this work are: (1) to examine further the case of two-dimensional variability in certain limits of scaling; (2) to compare analytical and numerical solutions; and (3) to extend the analysis to the case of two distinct fluids of different density.

\footnotetext{
*danders1@gmu.edu

†rmm@amath.unc.edu

${ }^{\ddagger}$ casey_miller@unc.edu
} 


\section{SINGLE FLUID GRAVITY CURRENT: HETEROGENEOUS POROUS MEDIA}

\subsection{Governing Equations}

The equations governing the evolution of a single-fluid gravity current in a porous media with permeability function $K_{c}(x, z)$ that varies in space are given by the variable coefficient elliptic problem

$\nabla \cdot\left[K_{c}(x, z) \nabla \phi\right]=0$,

where $\phi=p /(\rho g)+z, p$ is the pressure and $g$ is the gravitational acceleration in the negative $z$ direction. This equation is subject to boundary conditions corresponding to an impermeable boundary and zero pressure at the free boundary

$\begin{aligned} \frac{\partial \phi}{\partial z} & =0, \quad \text { at } z=0, \\ \phi & =h(x, t), \quad \text { at } z=h(x, t) .\end{aligned}$

The interface $h(x, t)$ evolves according to the kinematic boundary condition

$\frac{\partial h}{\partial t}=\frac{\partial}{\partial x} \int_{0}^{h} K_{c}(x, z) \frac{\partial \phi}{\partial x} d z$.

This equation is subject to symmetry and contact boundary conditions

$$
\begin{aligned}
\frac{\partial h}{\partial x} & =0, & & \text { at } x=0, \\
h & =0, & & \text { at } x=R(t),
\end{aligned}
$$

where $R$ is the radial position of the contact line. It follows that the contact line $x=R(t)$ moves with the horizontal fluid velocity $d R / d t=u(R, t)$. See AMM for further details.

\subsection{Thin Geometry Limit}

In the limit of a thin geometry $\delta=H / L \ll 1$, where the vertical length scale of the fluid region $H$ is much smaller than the horizontal length scale $L$, the evolution equation for $h$ can be decoupled from the elliptic problem so that $h$ satisfies the following partial differential equation

$\frac{\partial h}{\partial t}=\frac{\partial}{\partial x}\left[\frac{\partial h}{\partial x} \int_{0}^{h} K_{c}(x, \delta z) d z\right]$

This equation is subject to suitable boundary conditions as in (5) and (6). When $K_{c}$ is constant, this equation admits a similarity solution that can be written down analytically (see AMM for details). For some variable permeability functions, it may be appropriate to approximate $K_{c}(x, \delta z)$ by $K_{c}(x, 0)$; such would be the case if there was relatively slow variation of the permeability in the vertical direction or if the geometry of the fluid was so thin as to only sample a section of the media that was effectively of uniform permeability with respect to vertical variation. We shall describe other scenarios in the sections that follow.

\subsection{Special Limit of Two-Dimensional Periodicity}

AMM examined equations (1)-(6) for $K_{c}=K_{c}(x / \epsilon, z / \epsilon)$ where $\epsilon$ is a small parameter measuring the length scale associated with the permeability variations in the porous media. They applied homogenization techniques in the limit $\epsilon \rightarrow 0$ and found that in the fully two-dimensional case (non-thin geometry) the elliptic problem can be reduced to one with constant coefficients, and that in the thin geometry limit a single constant effective permeability appears in the evolution equation for the interface [see equation (18)]. However, in order to determine these coefficients one must generally solve cell problems (22) numerically. Special forms of $K_{c}$ allow exact solutions to be found (e.g. see Holmes[5]). In this section 
we describe another special case in which homogenization techniques can be applied in the thin limit to explicitly identify the effective permeability coefficient.

Here we are interested the situation where $K_{c}=K_{c}\left(x / \epsilon, z / \epsilon^{2}\right)$; that is, there is rapid variation in the permeability function that occurs on a length scale of $O(\epsilon)$ in the horizontal direction and on a length scale of $O\left(\epsilon^{2}\right)$ in the vertical direction. Further, we are interested in the thin-geometry limit where $\delta$ represents the ratio of vertical to horizontal extent of the fluid region as described above. We assume that $\delta=O(\epsilon)$ and, in fact, for ease of exposition we take $\delta=\epsilon$. In this setting the permeability variations in the vertical and horizontal directions appear on the same scale in the rescaled coordinates of the thin geometry.

In order to proceed we introduce the rescaled variables $z=\delta z^{\prime}, X=x / \epsilon$ and $Z^{\prime}=z^{\prime} / \epsilon$. We then proceed with a multiscale expansion by formally taking

$$
\frac{\partial}{\partial x} \rightarrow \frac{\partial}{\partial x}+\frac{1}{\epsilon} \frac{\partial}{\partial X}, \quad \frac{\partial}{\partial z} \rightarrow \frac{1}{\delta}\left(\frac{\partial}{\partial z^{\prime}}+\frac{1}{\epsilon} \frac{\partial}{\partial Z^{\prime}}\right) .
$$

Substituting this into equation (1) gives

$$
\left(\frac{\partial}{\partial x}+\frac{1}{\epsilon} \frac{\partial}{\partial X}\right)\left[K_{c}\left(X, Z^{\prime}\right)\left(\frac{\partial \phi}{\partial x}+\frac{1}{\epsilon} \frac{\partial \phi}{\partial X}\right)\right]+\frac{1}{\epsilon^{2}}\left(\frac{\partial}{\partial z^{\prime}}+\frac{1}{\epsilon} \frac{\partial}{\partial Z^{\prime}}\right)\left[K_{c}\left(X, Z^{\prime}\right)\left(\frac{\partial \phi}{\partial z^{\prime}}+\frac{1}{\epsilon} \frac{\partial \phi}{\partial Z^{\prime}}\right)\right]=0 .
$$

We introduce an expansion in $\epsilon$ of the form

$\phi=\phi_{0}+\epsilon \phi_{1}+\epsilon^{2} \phi_{2}+\epsilon^{3} \phi_{3}+\ldots$

One can proceed with an expansion of the equations under the assumption that each $\phi_{i}$ depends on the variables $x, z^{\prime}, X$ and $Z^{\prime}$. However, based on the results of AMM and the expectation in the thin geometry that the dependence of $\phi$ on the vertical coordinate will not occur until higher orders, we make the following ansatz: $\phi_{0}=\phi_{0}(x), \phi_{1}=\phi_{1}(x, X), \phi_{2}=\phi_{2}\left(x, z^{\prime}, X\right)$ and $\phi_{i}=\phi_{i}\left(x, z^{\prime}, X, Z^{\prime}\right)$ for $i>2$. It can be verified that the $O\left(\epsilon^{-4}\right), O\left(\epsilon^{-3}\right)$ and $O\left(\epsilon^{-2}\right)$ expansions of equation (9) are identically satisfied under these assumptions.

The $O\left(\epsilon^{-1}\right)$ terms in equation (9) are

$$
\frac{\partial}{\partial Z^{\prime}}\left[K_{c}\left(\frac{\partial \phi_{2}}{\partial z^{\prime}}+\frac{\partial \phi_{3}}{\partial Z^{\prime}}\right)\right]+\frac{\partial}{\partial X}\left[K_{c}\left(\frac{\partial \phi_{0}}{\partial x}+\frac{\partial \phi_{1}}{\partial X}\right)\right]=0 .
$$

Averaging this in $Z^{\prime}$ and then integrating in $X$ gives

$$
\left\langle K_{c}\right\rangle_{Z^{\prime}}\left(\frac{\partial \phi_{0}}{\partial x}+\frac{\partial \phi_{1}}{\partial X}\right)=c(x)
$$

where $c(x)$ is a function of $x$ only (in particular it is independent of $X$ ). Dividing by $\left\langle K_{c}\right\rangle_{Z^{\prime}}$ and averaging in $X$, assuming that $\phi_{1}$ is periodic in $X$, shows that

$c(x)=\left\langle\left\langle K_{c}\right\rangle_{Z^{\prime}}^{-1}\right\rangle_{X}^{-1} \frac{\partial \phi_{0}}{\partial x}$.

Therefore,

$$
\left\langle K_{c}\right\rangle_{Z^{\prime}}\left(\frac{\partial \phi_{0}}{\partial x}+\frac{\partial \phi_{1}}{\partial X}\right)=\left\langle\left\langle K_{c}\right\rangle_{Z^{\prime}}^{-1}\right\rangle_{X}^{-1} \frac{\partial \phi_{0}}{\partial x}
$$

The problem is not complete until we examine the boundary conditions. As described in AMM, our approach with the interface evolution equation (4) when applying homogenization techniques in this case is to formally 'pre-average' terms in the boundary condition that depend on $Z^{\prime}$ in order to eliminate the fast variation in the vertical direction before applying the boundary condition. This was justified in 
4

AMM for the case where $K_{c}=K_{c}(z / \epsilon)$. We provide further justification here by comparison of these results to direct numerical simulations (see next section). The pre-averaged boundary condition is

$\frac{\partial h}{\partial t}=\frac{\partial}{\partial x} \int_{0}^{h}\left\langle K_{c} \frac{\partial \phi}{\partial x}\right\rangle_{Z^{\prime}} d z^{\prime}$.

Consistent with our expansion for $\phi$, we take $h=h_{0}(x)+\epsilon h_{1}(x, X)+\ldots$ Furthermore, we note that since $\phi_{0}=\phi_{0}(x)$ the boundary condition (3) implies that $\phi_{0}=h_{0}$. Expanding equation (15) in multi-scales gives at $O(1)$

$$
\begin{aligned}
\frac{\partial h_{0}}{\partial t} & =\frac{\partial}{\partial x}\left[h_{0}\left\langle K_{c}\left(\frac{\partial \phi_{0}}{\partial x}+\frac{\partial \phi_{1}}{\partial X}\right)\right\rangle_{Z^{\prime}}\right] \\
& +\frac{\partial}{\partial X}\left[h_{1}\left\langle K_{c}\left(\frac{\partial \phi_{0}}{\partial x}+\frac{\partial \phi_{1}}{\partial X}\right)\right\rangle_{Z^{\prime}}\right] \\
& +\frac{\partial}{\partial X}\left[h_{0}\left\langle K_{c}\left(\frac{\partial \phi_{1}}{\partial x}+\frac{\partial \phi_{2}}{\partial X}\right)\right\rangle_{Z^{\prime}}\right] .
\end{aligned}
$$

Averaging this in $X$, using equation (14) and $\phi_{0}=h_{0}$ gives

$\frac{\partial h_{0}}{\partial t}=\left\langle\left\langle K_{c}\right\rangle_{Z^{\prime}}^{-1}\right\rangle_{X}^{-1} \frac{\partial}{\partial x}\left[h_{0} \frac{\partial h_{0}}{\partial x}\right]$,

which indicates that the effective permeability is $\left\langle\left\langle K_{c}\right\rangle_{Z^{\prime}}^{-1}\right\rangle_{X}^{-1}$ which is the harmonic average in $X$ of the arithmetic average in $Z^{\prime}$ of the permeability function.

We compare this result with the more general result derived in AMM where for the thin geometry limit with permeability function $K_{c}(x / \epsilon, z / \epsilon)$, the problem for the interface position was reduced to solving

$\frac{\partial h_{0}}{\partial t}=K_{e f f} \frac{\partial}{\partial x}\left[h_{0} \frac{\partial h_{0}}{\partial x}\right]$

with effective permeability function $K_{\text {eff }}=\alpha-\beta^{2} / \gamma$. The coefficients $\alpha, \beta$ and $\gamma$ were given by

$\alpha=\left\langle\left\langle K_{c}\right\rangle\right\rangle-\left\langle\left\langle K_{c}\left|\nabla \theta_{1}\right|^{2}\right\rangle\right\rangle$,

$\beta=-\left\langle\left\langle K_{c} \nabla \theta_{1} \cdot \nabla \theta_{2}\right\rangle\right\rangle$,

$\gamma=\left\langle\left\langle K_{c}\right\rangle\right\rangle-\left\langle\left\langle K_{c}\left|\nabla \theta_{2}\right|^{2}\right\rangle\right\rangle$

Here the functions $\theta_{1}$ and $\theta_{2}$ satisfied the two cell problems

$\mathcal{L} \theta_{1}=-\frac{\partial K_{c}}{\partial X}, \quad \mathcal{L} \theta_{2}=-\frac{\partial K_{c}}{\partial Z}$

where $Z=z / \epsilon$ with periodic boundary conditions on $X \in[0,1], Z \in[0,1]$, where the linear operator $\mathcal{L}$ was given by

$\mathcal{L}=\frac{\partial}{\partial X}\left(K_{c} \frac{\partial}{\partial X}\right)+\frac{\partial}{\partial Z}\left(K_{c} \frac{\partial}{\partial Z}\right)$.

Additionally, $\langle\langle\cdot\rangle\rangle=\int_{0}^{1} \int_{0}^{1} \cdot d X d Z$. An iterative solution of the above cell problem with $Z=\delta Z^{\prime}$ and $\delta \rightarrow 0$ also reveals that $K_{\text {eff }}=\left\langle\left\langle K_{c}\right\rangle_{Z^{\prime}}^{-1}\right\rangle_{X}^{-1}$ but one must take the precaution that the result in AMM was derived for the case $K_{c}(x / \epsilon, z / \epsilon)$ whereas the result derived in this section applied specifically for the thin limit with $K_{c}\left(x / \epsilon, z / \epsilon^{2}\right)$. We further note that this particular formula for $K_{\text {eff }}$ is exact for permeabilities whose heterogeneity admit multiplicative factorization $K_{c}(X, Z)=H(X) G(Z)$ (see e.g. Holmes $[5])$ and agrees with the slender limit calculation just presented, as well as with the iterated homogenization. More generally, the cell problems require numerical inversion.

The results of this section can be compared to numerical simulations of the original slender limit equations. We show this comparison below. 


\subsection{Numerical Simulations}

In the limit of a thin geometry, the evolution equation for $h$ is given by equation (7) subject to the boundary conditions (5) and (6). The contact line condition

$\frac{d R}{d t}=u(R, t)=-\left.K_{c}(R / \epsilon, 0) \frac{\partial h}{\partial x}\right|_{x=R}$.

is also directly enforced. The initial conditions are $h(x, 0)=A_{H}\left(1-x^{2}\right)$ and $R(0)=1$ where $A_{H}$ is a given constant. We consider the permeability function

$K_{c}\left(x, z^{\prime}\right)=1+A \cos \left(\frac{2 \pi x}{\epsilon}\right)+B \cos \left(\frac{2 \pi z^{\prime}}{\epsilon}\right)$

with $\epsilon=0.5, A=B=0.2$. Here $\left\langle\left\langle K_{c}\right\rangle_{Z^{\prime}}^{-1}\right\rangle_{X}^{-1}=0.9797$. We solve this system numerically using a finite difference approach with second-order accurate central differencing in space and a backward Euler implicit time stepping routine. For more details on the numerical method, see 'Approach B' described in AMM.

Figure 1 shows the direct numerical simulation of the slender geometry model calculated with the variable permeability function (25) [solid curve] and the numerical solution of equation (17) with the effective permeability [dashed curve] as noted above. The leading order homogenization result clearly captures the global behavior of the gravity current. The small variation away from this global behavior in response to the local permeability can be observed in the full numerical solution. The close-up in the lower plot shows that in regions where the permeability is relative high and the fluid relatively mobile, the actual interface position is flatter on average than the homogenized solution. Where the permeability is relatively low and the fluid is less mobile, the actual interface position is steeper on average than the homogenized solution.

\section{TWO LAYER GRAVITY CURRENT:}

As in the above description, AMM considered only a single fluid model. In this section we develop an analogous two-fluid model and show how it can be related back to the single fluid models previously discussed, thereby extending the applicability and interpretation of the single fluid model.

Consider a slumping of mound of fluid with density $\rho_{1}$ in a porous media along an impermeable boundary at $z=0$. The height of this layer is given by $z=h(x, t)$. A second fluid with density $\rho_{2}$ surrounds fluid 1 . The second fluid has an interface with air at a height $z=H$ in the porous layer. Fluid 1 occupies the region $0 \leq x \leq R(t)$ (assumed symmetric about $x=0$ ) and $0 \leq z \leq h(x, t)$ where $R(t)$ represents the contact line at the leading edge of the gravity current. Fluid 2 is assumed to extend infinitely in the horizontal direction. We are interested in the case where the overall layer thickness $H$ is much greater than the maximum value of $h$, and consequently assume that $H$ is constant.

In fluid 1 , the governing equations are

$$
\begin{aligned}
u_{1} & =-K_{1} \frac{\partial \phi_{1}}{\partial x} \\
w_{1} & =-K_{1} \frac{\partial \phi_{1}}{\partial z} \\
0 & =\frac{\partial u_{1}}{\partial x}+\frac{\partial w_{1}}{\partial z},
\end{aligned}
$$

where $\phi_{1} \equiv p_{1} /\left(\rho_{1} g\right)+z$ and the fluid velocity has horizontal and vertical components $u_{1}$ and $w_{1}$. Similarly, in fluid 2 the governing equations are

$$
u_{2}=-K_{2} \frac{\partial \phi_{2}}{\partial x}
$$



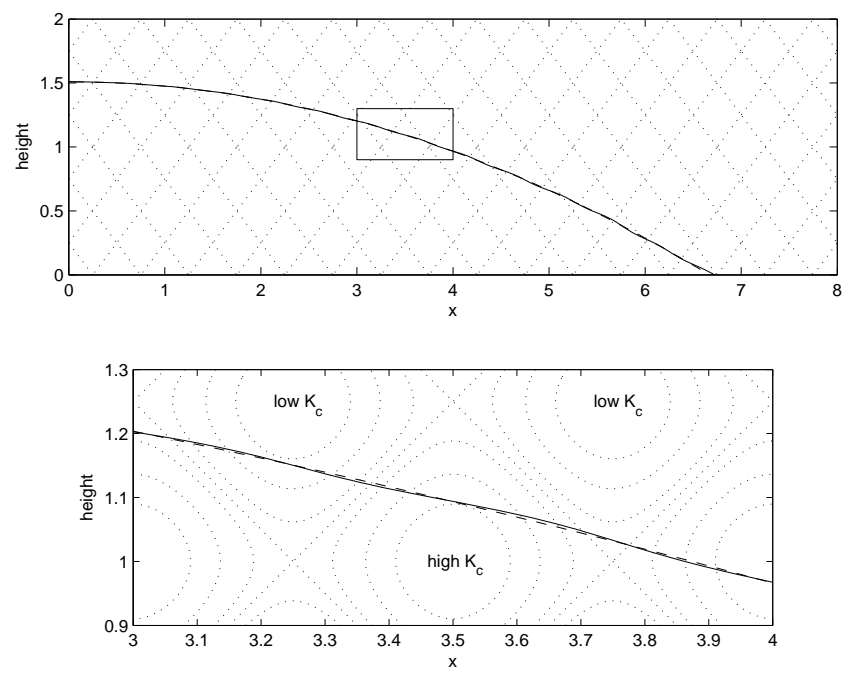

Figure 1. The upper figure shows the background permeability structure and the interface position calculated numerically using the thin geometry evolution equation (solid line) and the leading order homogenized solution (dashed line). A close-up view of the region in the box in the upper figure is shown in the lower figure.

$$
\begin{aligned}
w_{2} & =-K_{2} \frac{\partial \phi_{2}}{\partial z}, \\
0 & =\frac{\partial u_{2}}{\partial x}+\frac{\partial w_{2}}{\partial z},
\end{aligned}
$$

where $\phi_{2} \equiv p_{2} /\left(\rho_{2} g\right)+z$ and the fluid velocity has horizontal and vertical components $u_{2}$ and $w_{2}$. The permeability functions $K_{1}$ and $K_{2}$ are assumed for simplicity to be constants.

The boundary condition applied at $z=H$ is that $p_{2}=0$ (constant atmospheric pressure taken to be zero without loss of generality). The boundary conditions along the impermeable base at $z=0$ are that $w_{2}=0$ for $x>R(t)$ and $w_{1}=0$ for $0 \leq x \leq R$. At the fluid/fluid interface we have $p_{1}=p_{2}$ and the mass balance conditions $\vec{u}_{1} \cdot \hat{n}=\vec{u}_{2} \cdot \hat{n}=\vec{u}_{I} \cdot \hat{n}$, where $\vec{u}_{1}=\left(u_{1}, w_{1}\right), \vec{u}_{2}=\left(u_{2}, w_{2}\right)$ and $\vec{u}_{I}$ is the velocity of the interface. We express these conditions as follows

$$
\begin{aligned}
\phi_{2}=H, & \text { at } z=H, \\
\frac{\partial \phi_{2}}{\partial z}=0 & \text { at } z=0 \text { for } x>R(t), \\
\frac{\partial \phi_{1}}{\partial z}=0 & \text { at } z=0 \text { for } 0 \leq x \leq R(t) .
\end{aligned}
$$

The boundary conditions at the interface $z=h(x, t)$ can be expressed as

$$
\begin{aligned}
\rho_{1}\left(\phi_{1}-h\right) & =\rho_{2}\left(\phi_{2}-h\right), \\
-u_{1} h_{x}+w_{1} & =-u_{2} h_{x}+w_{2}=\frac{\partial h}{\partial t} .
\end{aligned}
$$

The geometry is assumed to be infinite in the horizontal direction so that $\phi_{2} \rightarrow H$ as $|x| \rightarrow \infty$. 


\subsection{Approximate Solution}

In this section we describe a solution to the above equations that applies when fluid 1 can be considered 'thin' [its horizontal extent is much larger than its maximum height]. In this scenario the governing equations in fluid 1 reduce to

$$
\begin{aligned}
u_{1} & =-K_{1} \frac{\partial \phi_{1}}{\partial x} \\
0 & =-K_{1} \frac{\partial \phi_{1}}{\partial z} \\
w_{1} & =-\int_{0}^{z} \frac{\partial u_{1}}{\partial x} d z
\end{aligned}
$$

to leading order. This implies that $\phi_{1}=\phi_{1}(x, t)$ is independent of $z$. Then, from equation (35) we have

$$
\phi_{1}(x, t)=h+\frac{\rho_{2}}{\rho_{1}}\left[\phi_{2}(x, h, t)-h\right] .
$$

Equation (40) implies that

$$
\frac{\partial \phi_{1}}{\partial x}=\left(1-\frac{\rho_{2}}{\rho_{1}}\right) h_{x}+\frac{\rho_{2}}{\rho_{1}}\left[\frac{\partial \phi_{2}}{\partial x}(x, h, t)+h_{x} \frac{\partial \phi_{2}}{\partial z}(x, h, t)\right] .
$$

The fluid/fluid interface $h(x, t)$ evolves according to

$$
\frac{\partial h}{\partial t}=\frac{\partial}{\partial x} \int_{0}^{h} K_{1} \frac{\partial \phi_{1}}{\partial x} d z=\frac{\partial}{\partial x}\left[K_{1} h \frac{\partial \phi_{1}}{\partial x}\right]
$$

which can be solved once suitable information about derivatives of $\phi_{2}$ is known.

The mass balance boundary condition (36) can be expressed as

$\frac{\partial}{\partial x} \int_{0}^{h} u_{1} d z=\frac{\partial}{\partial x} \int_{H}^{h} u_{2} d z$.

Integrating with respect to $x$ and noting that $u_{1}=u_{2}=0$ along the line of symmetry $x=0$ gives

$\int_{0}^{h} K_{1} \frac{\partial \phi_{1}}{\partial x} d z=\int_{H}^{h} K_{2} \frac{\partial \phi_{2}}{\partial x} d z$

Since $\phi_{1}$ is independent of $z$ we have

$K_{1} \frac{\partial \phi_{1}}{\partial x} h=\int_{H}^{h} K_{2} \frac{\partial \phi_{2}}{\partial x} d z$.

Using equation (41) in equation (45) leads to the expression

$K_{1} h\left\{\left(1-\frac{\rho_{2}}{\rho_{1}}\right) h_{x}+\frac{\rho_{2}}{\rho_{1}}\left[\frac{\partial \phi_{2}}{\partial x}(x, h, t)+h_{x} \frac{\partial \phi_{2}}{\partial z}(x, h, t)\right]\right\}=\int_{H}^{h} K_{2} \frac{\partial \phi_{2}}{\partial x} d z$,

which is effectively a boundary condition for $\phi_{2}$ at the interface $z=h$. This equation and $\phi_{2}(x, H, t)=H$ are the boundary conditions for the $\phi_{2}$ problem defined above.

A simple approximate solution to the $\phi_{2}$ problem can be obtained in the case where the layer thickness $H$ is much larger that the thickness of the gravity current. This is the deep layer case where $h / H \rightarrow 0$. Here, $\phi_{2}=H$ to leading order. Equation (46) is satisfied to leading order by the right-hand side vanishing [the left-hand side would enter at a higher order contributing to corrections to $\phi_{2}=H$ ]. In this limit equation (41) implies that

$$
\frac{\partial \phi_{1}}{\partial x}=\left(1-\frac{\rho_{2}}{\rho_{1}}\right) h_{x}
$$


$\frac{\partial h}{\partial t}=\left(1-\frac{\rho_{2}}{\rho_{1}}\right) \frac{\partial}{\partial x}\left[K_{1} h \frac{\partial h}{\partial x}\right]$.

Notice that in the limit $\rho_{2} / \rho_{1} \rightarrow 0$ we recover the single layer case. If $\rho_{2}=\rho_{1}$ the interface is stationary. If $\rho_{2}>\rho_{1}$ we have a slumping gravity current in reverse [i.e. it steepens]. This is effectively the same evolution equation considered in the single fluid problem [compare, for example, equation (18)] if time were rescaled to account for the density difference factor.

\section{CONCLUSIONS}

The following conclusions are drawn from this work:

1. We have shown that homogenization methods are useful for the description of nonlinear, free-surface problems involving the movement of dense brines in porous medium systems.

2. We have built upon previous homogenization results by considering a special two-dimensional case in a thin limit and validated our numerical results, adding further evidence for the validity of our formal "pre-averaging" of the boundary condition to obtain a closed, large scale evolution.

3. We have further derived an approximate solution for a two fluid case in which the interface between miscible fluids of contrasting densities evolve with time for a thin limit case.

4. Continued development and application of both leading order and first-order corrections based upon homogenization methods used to describe density dependent flow in heterogeneous porous medium systems appears both possible and worthwhile, with several additional extensions summarized for future work.

Our future plans involve further validating the homogenization techniques used here and in AMM for non-slender geometries, which requires directly solving the variable-coefficient elliptic problem. Our interest is in cases of layered permeabilities as well as two-dimensional periodic permeabilities. The latter, as noted above, generally requires the solution of cell problems in order to determine the necessary coefficients for the homogenized solutions although special cases, as described in the present work, will also be considered.

\section{REFERENCES}

1. C.T. Miller, G. Christakos, P.T. Imhoff, J.F. McBride, J.A. Pedit, and J.A. Trangenstein, "Multiphase flow and transport modeling in heterogeneous porous media: Challenges and approaches," Adv. Water Resources, 21(2) 77-120 (1998).

2. C.T. Miller, E.H. Hill III and M. Moutier, "Remediation of DNAPL-contaminated subsurface systems using density-motivated mobilization," Env. Sci. Tech. 34(4) 719-724 (2000).

3. E.H. Hill III, M. Moutier, J. Alfaro, and C.T. Miller, "Remediation of DNAPL pools using dense-brine barrier strategies," Env. Sci. Tech. 35(14) 3031-3039 (2001).

4. D.M. Anderson, R.M. McLaughlin \& C.T. Miller, The Averaging of Gravity Currents in Porous Media, Phys. Fluids 15 (2003) 2810-2829.

5. M.H. Holmes, Introduction to Perturbation Methods, (Springer, New York, 1995) pp. 236-237. 